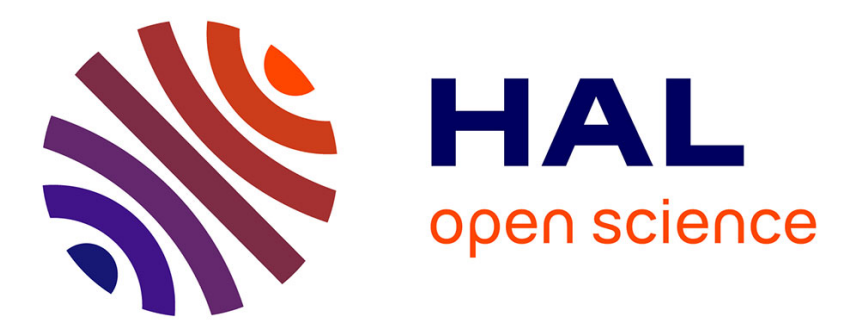

\title{
High-Tc-based Josephson-junctions and SQUIDs by mechanically induced growth disorder
}

\author{
M. Schmitt, A. Hadish, P. Wagner, Th. Becherer, H. Adrian
}

\section{To cite this version:}

M. Schmitt, A. Hadish, P. Wagner, Th. Becherer, H. Adrian. High-Tc-based Josephson-junctions and SQUIDs by mechanically induced growth disorder. Journal de Physique IV Proceedings, 1994, 04 (C6), pp.C6-217-C6-222. 10.1051/jp4:1994635 . jpa-00253130

\section{HAL Id: jpa-00253130 https://hal.science/jpa-00253130}

Submitted on 1 Jan 1994

HAL is a multi-disciplinary open access archive for the deposit and dissemination of scientific research documents, whether they are published or not. The documents may come from teaching and research institutions in France or abroad, or from public or private research centers.
L'archive ouverte pluridisciplinaire HAL, est destinée au dépôt et à la diffusion de documents scientifiques de niveau recherche, publiés ou non, émanant des établissements d'enseignement et de recherche français ou étrangers, des laboratoires publics ou privés. 


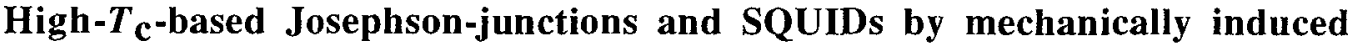 growth disorder
}

\author{
M. Schmitt, A. Hadish, P. Wagner, Th. Becherer and H. Adrian \\ Technische Hochschule, Institut für Festkörperphysik, Hochschulstraße 8, 64289 Darmstadt, Germany
}

\begin{abstract}
We have developped a simple method for the reproducible production of high$T_{c}$ based Josephson junctions and SQUIDs. By producing a very weak deformation into the substrate surface by a diamond needle before depostion of the thin high- $T_{c}-$ film, the film shows a growth disturbance in the region of the deformation with quite interesting features, resulting in a Josephson junction. The substrate deformation is characterized by Atomic Force Microscopy and the film growth over the deformation is examined by Scanning Electron Microscopy. The junctions produced by this method show RSJ-like behaviour in their current-voltage characteristics and Shapiro-steps at all temperatures between 4.2 and $89 \mathrm{~K}$, when exposed to microwave power. The $I_{c} R_{n}$-products are variable in a wide range and can be very high for properly chosen film thicknesses. SQUIDs produced by this method show voltage modulations due to dc-flux of up to $12 \mu \mathrm{V}$ peak to peak at 77K for low SQUIDperformance parameters $\beta \ll 1$. For $\beta \gg 1$ the modulations are between 3 and $5 \mu \mathrm{V}$ at $77 \mathrm{~K}$. Finally the noise in SQUIDs produced by this method is examined. It is shown that the noise power is in the region of that of step edge junctions.
\end{abstract}

\section{INTRODUCTION}

Due to the short coherence length in High- $T_{c}$-superconductors, there had to be developed new and sophisticated methods for the production of Josephson-junctions and SQUIDs with these materials. Step-edge-[1,6], biepitaxy-[2], bicristal-[3], ramp-type-[4], and SNS-junctions[5] are the most important ones. All these methods have in common that they are either unflexible in design or in their superconducting properties as critical current $I_{c}$ or the $I_{c} R_{n}$-product. The method introduced in this paper allows for the reproducible production of Josephson-junctions and SQUIDs in a wide paramter range. It consits of a very weak deformation made into the substrate surface by a diamond needle before film deposition. The method allows for high flexibility in design.

\section{PREPARATION}

\subsection{Preparation and Characterization of the Deformation}

The deformation of the substrate surface is produced by a commercially available diamond needle. When pressing the needle onto the substrate with a pressure of $2.5-250 \mathrm{GPa}$, the substrate is moved linearly with a velocity of $0.3-0.5 \mathrm{~mm} / \mathrm{sec}$.

Atomic Force Microscopy- (AFM-) examinations show, that the deformation has a width of about $1 \mu \mathrm{m}$ and a depth of 30 to $70 \mathrm{~nm}$. Enhanced edges on both sides are generated, which are part of the 


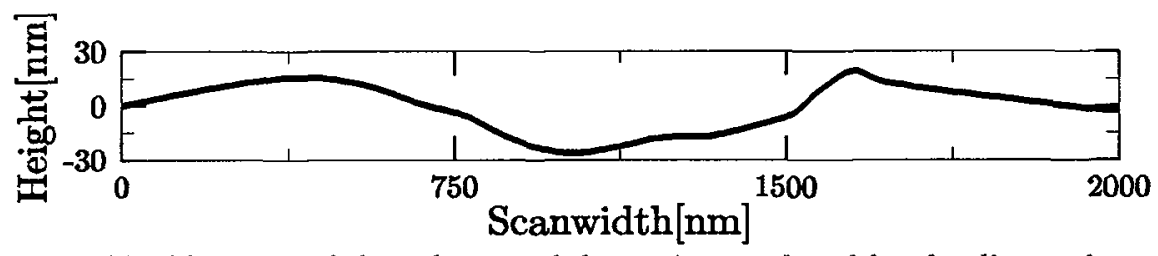

Figure 1: AFM-linescan of the substrate deformation produced by the diamond needle.

deformation, as they cannot be removed by chemical or mechanical cleaning. A typical AFM-result is shown in figure 1 .

As the width to depth ratio of the deformation is much larger than 10:1, the resulting Josephsonjunctions are not of the step-edge type, as for these a tilt angle of at least $45^{\circ}$ is required[6]. $\mathrm{SrTiO}_{3}$ as well as $\mathrm{NdGaO}_{3}$ were used as substrate-materials. With both materials equal properties of the deformation and of the later deposited high- $T_{c}$-film were observed.

\subsection{Preparation and Characterization of the thin Film}

The thin $\mathrm{YBa}_{2} \mathrm{Cu}_{3} \mathrm{O}_{7}$-film is deposited onto the substrates with the previously described deformation by dc-sputtering. We sputter from a stoichiometric and planar target in on-axis geometry in pure oxygen atmosphere and at high pressure[7]. The thin $\mathrm{YBa}_{2} \mathrm{Cu}_{3} \mathrm{O}_{7}$-films grown by this method onto substrates without deformation have a critical temperature $T_{\mathrm{c}}$ of $90-93 \mathrm{~K}$ in ac-susceptibility measurements with a sharp transition of smaller than $1 \mathrm{~K}$. X-ray diffraction measurements in BraggBrentano-geometry show that the films are completely c-axis oriented (only (00l)-peaks are seen) and that there is no indication for any other phases. The critical current denstity $j_{c}$ has a value of 4 to $6 \cdot 10^{6} \mathrm{~A} / \mathrm{cm}^{2}$ at $77 \mathrm{~K}$ and of 3 to $4 \cdot 10^{7} \mathrm{~A} / \mathrm{cm}^{2}$ at $4.2 \mathrm{~K}$.

\subsection{Scanning Electron Microscopy (SEM) of the Growth Disturbance}

The films deposited onto substrates with deformation were examined by SEM. In figure 2a a SEMpicture of the film-growth over the substrate deformation is shown for a $40 \mathrm{~nm}$ thick film on $\mathrm{SrTiO}_{3}$. The width of the deformation is approximately $1 \mu \mathrm{m}$ and is indicated by the white lines. The film grows homogeniously over nearly the whole disturbed region by forming growth steps. Only in a very narrow area of a width of approximately $100 \mathrm{~nm}$ (shown by the arrows) the growth of the film is heavily disturbed. Over this region contact is established only by nanobridges of width between 150 and $250 \mathrm{~nm}$. Between these bridges there is no film-growth at all. It is assumed that the resulting Josephson contacts are due to these nanobridges.

As the nanobridges are smaller or equal both in width and in thickness than the magnetic penetration depth $\lambda$ in $\mathrm{YBa}_{2} \mathrm{Cu}_{3} \mathrm{O}_{7}$, a Josephson effect could appear due to coherent vortex movement in the bridges $[8,9]$ without the assumption of any other weak link. Then the current-voltage- (I-V-) characteristics should have flux flow behaviour with $V \propto I^{\alpha}$. Additionally the dependence of the appearing Shapiro-steps on microwave power should behave in a totally other way than predicted by the Resistively Shunted Junction- (RSJ-) model[10], and the current-steps should not oscillate with increasing power. This is not the case as will be seen in the next section.

If the film thickness is more than approximately $80-100 \mathrm{~nm}$, the disturbance begins to be overgrown by $\mathrm{YBa}_{2} \mathrm{Cu}_{3} \mathrm{O}_{7}$ and the nanobridges become larger in width, thickness and number. In figure $2 \mathrm{~b}$ the growth disturbance of a film with a thickness of $80 \mathrm{~nm}$ is shown. The heavily disturbed region of film growth has nearly disappeared and is hardly discernible. Only the $1 \mu \mathrm{m}$ wide deformation region can be observed (white lines). As a consequence the Josephson effect disappears. Therefore for the successful and reproducible preparation of Josephson junctions with the mechanically induced growth disorder, high quality ultrathin films with a controllable thickness between 10 and $80 \mathrm{~nm}$ are required. The dc-sputtering method provides these films with control of the film thickness via the deposition time. However, the danger of superconducting shortcuts is quite high in this type of 

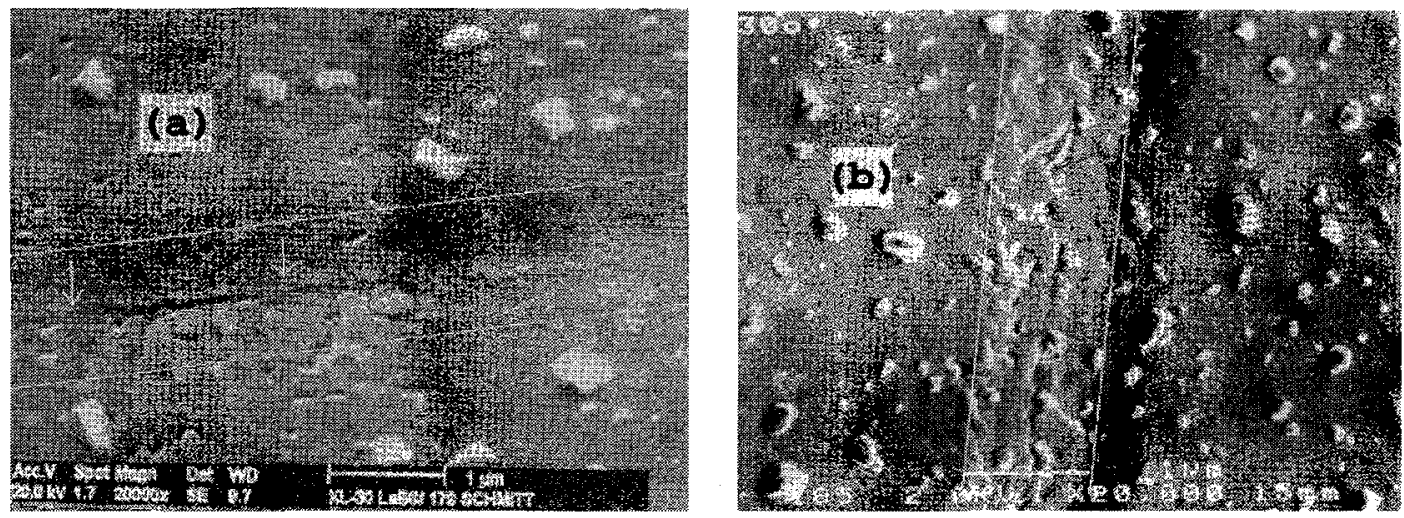

Figure 2: (a) SEM-picture of a structured film with a thickness of 40nm. Inbetween the white lines the substrate deformation is located. The arrows show the nanobridges crossing the approximately $100 \mathrm{~nm}$ wide heavily disturbed region. (b) SEM-picture of a film with a thickness of $80 \mathrm{~nm}$. The deformation made by the diamond needle can be well seen between the white lines, whereas the heavily disturbed region of film growth has nearly disappeared.

junction and cannot be excluded.

\section{PROPERTIES OF THE JOSEPHSON JUNCTIONS}

\subsection{Resistive Transitions of the Junctions}

In figure 3 the resistive transition $R(T)$ of a junction with a film thickness of $60 \mathrm{~nm}$ is shown. It can be seen that the film properties did not suffer due to the very small film thickness, as $T_{c}$ is as high as $92 \mathrm{~K}$. In the insert a small foot in the resistive transition is observable. Ambegaokar und Halperin $(\mathrm{AH})[11]$ could relate the suppression in the critical current of Josephson junctions for small currents to Thermally Activated Phase Slippage (TAPS). In this model the phase difference over the junction can be statistically thermally activated and slips by a value of $2 \pi$. The dependence of the mean additional resistance $R_{p}$ due to TAPS is given by $\mathrm{AH}$ :

$$
R_{p} / R=1 /\left(J_{\circ}(\gamma / 2)\right)^{2} ; \quad \gamma=h I_{c}(T) / 2 \pi e k_{B} T
$$

with $J_{o}$ the modified Bessel-function. Gross et al.[12] could fit their data for $\mathrm{YBa}_{2} \mathrm{Cu}_{3} \mathrm{O}_{7}$-grain boundary Josephson junctions with equation 1 and found a $\gamma$-value of 1350 , assuming a temperature dependence of the critical current near $T_{c}$ as

$$
I_{c}(T)=I_{c}(0) \cdot\left(1-T / T_{c}\right)^{2}
$$

We are able to fit our data with the same assumptions satisfactory (see fig. 3) and get $\gamma=26000$, which is more than one order of magnitude higher than found by Gross. This implies, if assumed that equation 2 were valid down to $T=0$, an $I_{c}(0)$-value of $50 \mathrm{~mA}$, corresponding to a critical current denstity $j_{c}(0)$ of $1 \cdot 10^{7} \mathrm{~A} / \mathrm{cm}^{2}$. In reality, $j_{c}$ is $5 \cdot 10^{5} \mathrm{~A} / \mathrm{cm}^{2}$ at $4 \mathrm{~K}$ and $4.5 \cdot 10^{4}-1 \cdot 10^{5} \mathrm{~A} / \mathrm{cm}^{2}$ at $77 \mathrm{~K}$, which is still very high in comparison to other junction types.

\subsection{Properties of the I-V-curves}

As can be seen from fig. 4, the I-V-characteristics are RSJ-like and non-hysteretic (McCumberparamter $\beta_{c}<1$ ). For properly chosen film thickness the $I_{c} R_{n}$-products can be quite high. By varying the film thickness, the $I_{c} R_{n}$-product can be changed in several orders of magnitude, as the critical current of the junctions has a non-linear dependence on the film thickness. The I-V-curves can be fitted with the RSJ-model including thermal current noise. In the junction of fig. 4 a Gaussian distribution of the fluctuation currents with a FWHM of $0.04 \mathrm{~mA}$ was assumed, which is quite low 

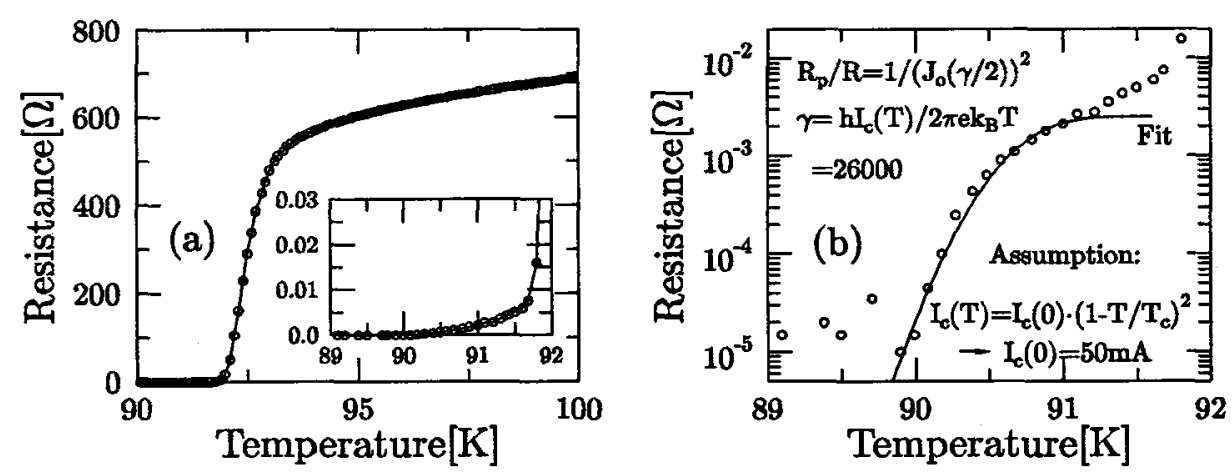

Figure 3: (a) Resistive transition of a junction with a film thickness of $60 \mathrm{~nm}$. In the insert a small foot can be seen, which can be fitted by the expression of Ambegaokar and Halperin for TAPS in the case of small currents. The fit is shown in (b).

in comparison with the critical current. However, the mean part of the I-V-curve could not be fitted by the RSJ-model and additionally, a considerably high excess-current had to be assumed for the fit. On the one hand this excess-current could be due to shortcuts, on the other hand, different types of junctions show such excess-currents, and their origin is still unknown.

\subsection{Influence of microwave power}

When exposed to microwave power, the junctions show Shapiro steps at all temperatures between 4.2 and $89 \mathrm{~K}$. In fig. $5 \mathrm{a}$ the I-V-curves of a $50 \mathrm{~nm}$ thick film on a $\mathrm{NdGaO}_{3}$ substrate at $4.2 \mathrm{~K}$ are shown for different microwave powers. The current steps oscillate with the microwave power. Due to the high excess-current, the oscillation does not behave as predicted by the RSJ-model. The first and second step begin to oscillate with increasing power before the width of the $0^{\text {th }}$ step is zero for the first time. For higher microwave powers the oscillation is RSJ-like. The step modulation in dependence of the microwave power is shown in figure $5 \mathrm{~b}$.

\subsection{Discussion}

As the I-V-curves are RSJ-like and there is a current-step modulation in dependence of the microwave power, we assume that the origin of the Josephson effect in our junctions is not due to coherent vortexmotion in the nanobridges, but is dominated by an additional weak link (grain boundary or SS'S-type device with reduced order parameter in the superconductor $S^{\prime}$ ), situated in the nanobridges.

For lower $T<77 \mathrm{~K}$, there often appear roundings in the I-V-curves, also not due to vortex motion but to more than one Josephson junction in line in the bridges which can well be identified in the derivatives of the I-V-curves.

\section{PROPERTIES OF THE DC-SQUIDS}

In fig.6a the voltage modulation of a dc-SQUID with a film thickness of $30 \mathrm{~nm}$ at $77 \mathrm{~K}$ and a SQUIDperformance parameter $\beta=2 L I_{c} / \Phi_{o}=3[13]$ is shown in dependence of the bias current through the SQUID. The inductance $L$ of the SQUID with a $10 \times 20 \mu \mathrm{m}^{2}$-hole is assumed to be $20 \mathrm{pH}$ and the critical current $I_{c}(77 \mathrm{~K})$ of the single junction (width $10 \mu \mathrm{m}$ ) is $0.15 \mathrm{~mA}$, corresponding to $j_{c}(77 K)=$ $4.5 \cdot 10^{4} \mathrm{~A} / \mathrm{cm}^{2}$.

The modulation depth is between $3-5 \mu \mathrm{V}$ peak to peak $\left(d V / d \Phi=9\right.$ to $\left.15 \mu \mathrm{V} / \Phi_{o}\right)$ for SQUIDs in the high $\beta$-limit. For lower temperatures $T<77 \mathrm{~K}$ the critical current increases by a factor of 5 to 10 , so that the high $\beta$-limit for the critical current modulation $\left(\Delta I_{c}=\Phi_{o} / L\right)$ is reached. In the low $\beta$-limit $(\beta \ll 1)$, peak to peak voltage modulations of up to $12 \mu \mathrm{V}$ could be reached at $77 \mathrm{~K}$, corresponding 

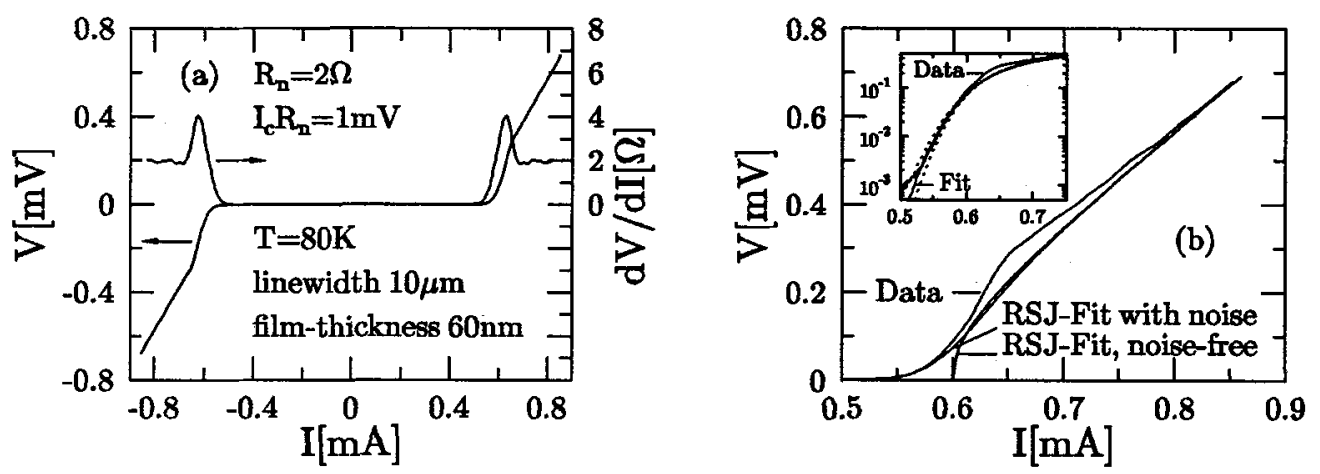

Figure 4: (a) I-V-curve of a junction with a film thickness of $60 \mathrm{~nm}$ at $T=80 \mathrm{~K}$ and its derivative. (b) Fit with the RSJ-model including thermal current fluctuations and the fit with the noise-free RSJ-model. The dotted lines in the insert are fits to Gaussian current distributions with FWHM of $0.04 \mathrm{~mA} \pm 10 \%$.
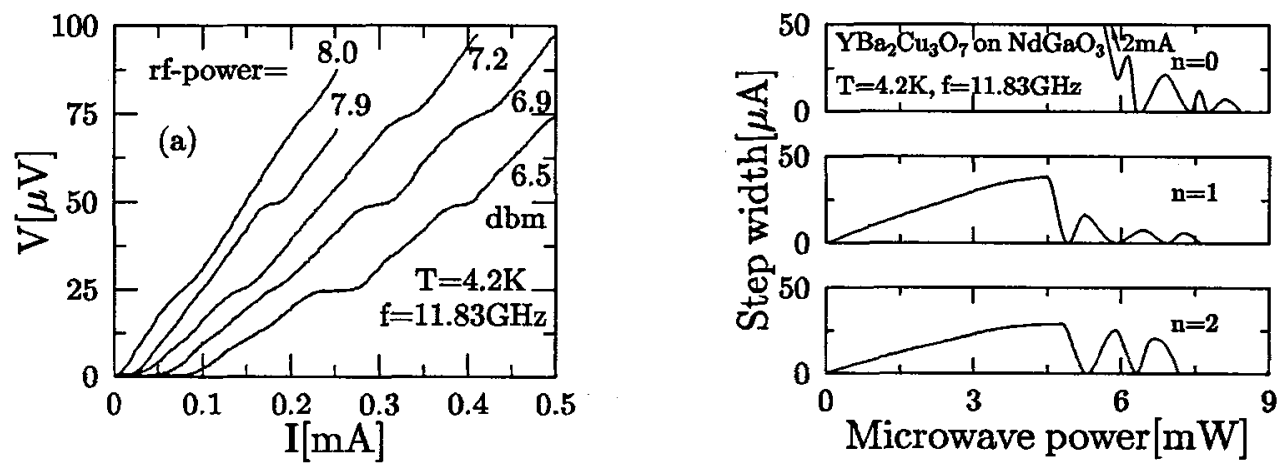

Figure 5: (a) I-V-curves for different microwave powers at $4.2 \mathrm{~K}$ for a $50 \mathrm{~nm}$ thick film on a $\mathrm{NdGaO}_{3}$ substrate. (b) Step-modulation in dependence of the microwave power.
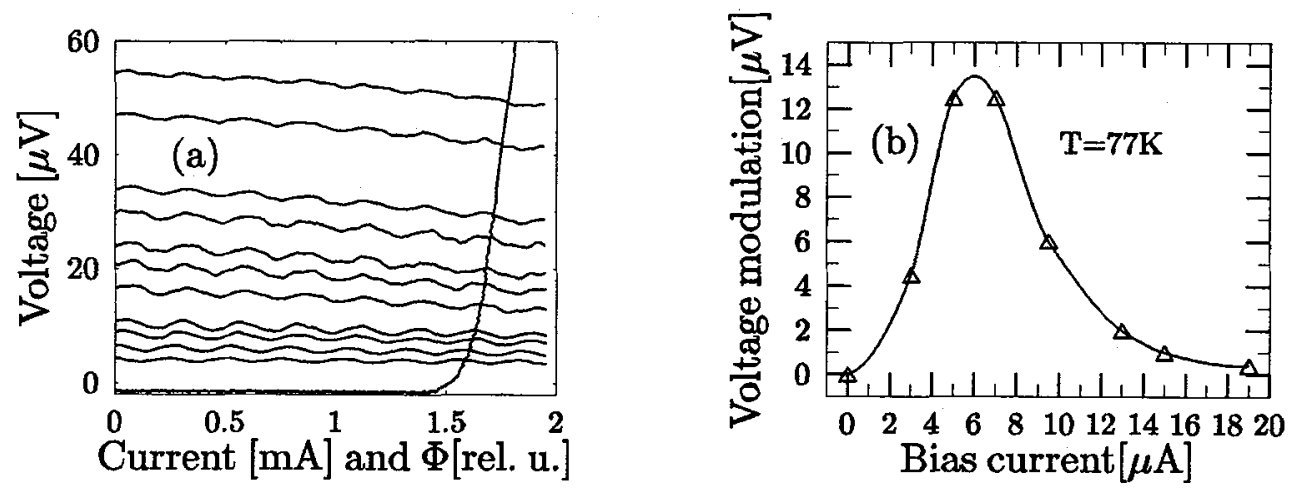

Figure 6: (a) Voltage modulation in dependence of magnetic flux of a SQUID at 77K with $\beta=3$ for different bias-currents. Additionally the I-V-curve is shown in the plot. (b) Voltage modulation of $a$ SQUID at $77 \mathrm{~K}$ with $\beta=0.025$ in dependence of the bias-current. 


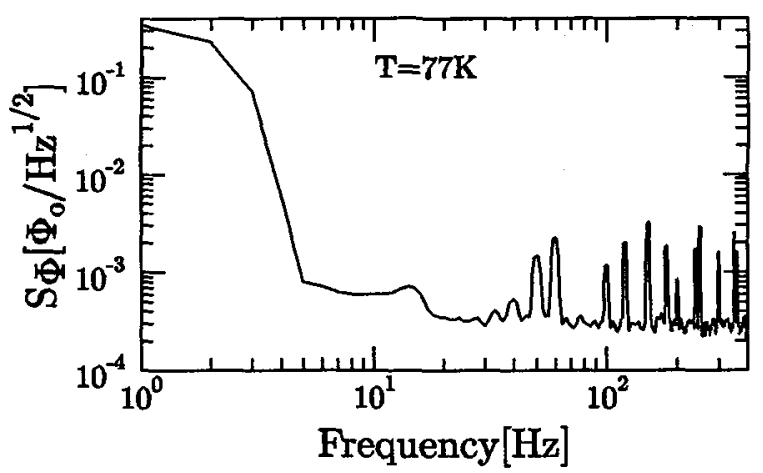

Figure 7: Flux noise in a dc-SQUID at 77K.

to $d V / d \Phi=36 \mu \mathrm{V} / \Phi_{\circ}$. The dependence of the voltage modulation of a SQUID with $\beta=0.025$ on the bias current is shown in fig. $6 \mathrm{~b}$.

In fig. 7 the flux noise for a SQUID at $77 \mathrm{~K}$ with linewidth of $10 \mu \mathrm{m}$ and a. $10 \times 20 \mu \mathrm{m}^{2}$ SQUID-hole is shown for frequencies between 1 and $400 \mathrm{~Hz}$. For frequencies higher than $5 \mathrm{~Hz}$ the noise is in the same range as it is for step-edge junctions $[14,15]$. For lower frequencies a significant increase of the noise power is observed, whose origin is not known. The peaks in the spectrum correspond to the line frequency of $50 \mathrm{~Hz}$ and some instruments working at $60 \mathrm{~Hz}$ and their harmonics.

As Josephson junctions for film thicknesses smaller than $20 \mathrm{~nm}$ are possible by the method of mechanically induced growth disorder, they provide the ideal combination with High- $T_{c}$ field effect devices, where ultrathin films are needed to have high field effects.

\section{References}

[1] R. W. Simon, J. F. Burch, K. P. Daly, W. D. Dozier, R. Hu, A. E. Lee, J. A. Luine, H. M. Manasevit, C. E. Platt, S. M. Schwarzbeck, D. St. John, M. S. Wire und M. J. Zani in R. D. McConnel und R. Nouf, Science and Technology in Thin Film Superconductors 2 (Plenum Press, New York, 1990) 549

[2] L. P. Lee, K. Char, M. S. Colclough und G. Zaharchuk, Appl. Phys. Lett. 59, 3051 (1991)

[3] D. Dimos, P. Chaudhari und J. Mannhart, Phys. Rev. B. 41, 4038 (1990)

[4] C. Stölzel, M. Siegel, G. Adrian, C. Krimmer, J. Söllner, W. Willems und H. Adrian, Appl. Phys. Lett. 63, 2970 (1993)

[5] M. S. Dilorio, S. Yoshizumi, K. Y. Yang, J. Zhang und M. Maung, Appl. Phys. Lett. 58, 2552 (1991)

[6] C. L. Jia, B. Kabius, K. Urban, K. Herrmann, G. J. Cui, J. Schubert, W. Zander, A. I. Braginski und C. Heiden, Physica C 175, 545 (1991)

[7] C. Tomé Rosa, G. Jakob, M. Maul, A. Walkenhorst, M. Schmitt, P. Wagner, P. Przyslupski und H. Adrian, Physica C 171, 231 (1990)

[8] L. G. Aslamazov und A. I. Larkin, Sov. Phys. JETP, 41, 381 (1975)

[9] K. K. Likharev, Sov. Phys. JETP 34, 906 (1972)

[10] D. E. McCumber, J. Appl. Phys. 39, 3113 (1968)

[11] V. Ambegaokar und B. I. Halperin, Phys. Rev. Lett. 22, 1364 (1969)

[12] R. Gross, P. Chaudhari, D. Dimos, A. Gupta und G. Koren, Phys. Rev. Lett. 64, 228 (1990)

[13] C. D. Tesche und J. Clarke, Jour. Low Temp. Phys. 29, 301 (1977)

[14] P. Seidel, E. Heinz, F. Schmidl, K. Zach, H. J. Köhler, H. Schneidewind, J. Borck, L. Dörrer, S. Linzen, and T. Köhler, IEEE Trans. Appl. Supercond. 3, 2353 (1993)

[15] H. K. Olsson, R. H. Koch, P. A: Nilsson, and A. E. Stepantsov, IEEE Trans. Appl. Supercond. 3, 2353 (1993) 